Data regarding vasopressor requirement as well as creatinine, bilirubin and aspartate aminotransferase levels are routinely collected before surgery. The RVFRS could, therefore, enable prediction of $\mathrm{RV}$ failure and mortality in candidates for LVAD surgery.

Original article Matthews JC et al. (2008) The right ventricular failure risk score: a pre-operative tool for assessing the risk of right ventricular assist device candidates. J Am Coll Cardiol 51:2163-2172

\section{Heparin contaminant causes adverse effects via the kallikrein system}

In January 2008, clusters of acute hypersensitivity reactions, characterized by hypotension and tachycardia, were reported in patients undergoing dialysis in the US. Investigations revealed that these reactions were caused by contaminated heparin samples, leading to two recalls of the drug. The contaminant was identified as an unusual oversulfated form of chondroitin sulfate (OSCS), but no biological link between OSCS and the adverse clinical events had been established. Kishimoto et al. have now determined that OSCS leads to the observed adverse events via activation of kallikrein, which can stimulate production of the vasoactive mediator bradykinin.

The authors showed that OSCS-contaminated heparin samples induced kallikrein amidolytic activity in human plasma at clinically relevant concentrations of heparin. Purified and synthetic OSCS also caused kallikrein activation, whereas uncontaminated heparin had no effect on the kallikrein system. Furthermore, contaminated heparin induced generation of the complement protein fragments $\mathrm{C} 3 \mathrm{a}$ and $\mathrm{C} 5 \mathrm{a}$, which are potent anaphylatoxins. Experiments conducted using factor-XII-depleted plasma suggest that OSCS generates these fragments by a pathway that is dependent on kallikrein activation through factor XII.

In in vivo studies, two out of six pigs that were injected with contaminated heparin samples displayed hypotension. Importantly, rapid induction of kallikrein activity was observed in all six pigs, even when no changes in blood pressure occurred.

The authors conclude that an in vitro assay for kallikrein amidolytic activity can enable easy screening for OSCS and could help to protect the global heparin supply from further contamination.

Original article Kishimoto TK et al. (2008) Contaminated heparin associated with adverse clinical events and activation of the contact system. N Engl J Med 358: 2457-2467

\section{Aprotinin is not recommended in patients undergoing high-risk cardiac surgery}

Randomized trials have demonstrated that three antifibrinolytic agents - the serine protease inhibitor aprotinin and the lysine analogs aminocaproic acid and tranexamic acid-reduce the need for blood transfusion in cardiac surgery. The data do not, however, support the definitive use of any one drug over the others. The Blood Conservation Using Antifibrinolytics in a Randomized Trial (BART) investigators aimed to establish whether aprotinin is superior to the other two agents in minimizing massive bleeding in patients undergoing high-risk procedures.

In this multicenter, blinded, randomized study, adult patients who were scheduled to undergo high-risk cardiac surgery, such as aortic procedures, valve replacement, or repeat CABG surgery, were randomly assigned to receive aprotinin $(n=780)$, aminocaproic acid $(n=780)$, or tranexamic acid $(n=770)$ during their operation. At 30 days after surgery, 261 $(11.2 \%)$ patients had experienced the primary end point of massive bleeding. There was a nonsignificant trend towards lower rates of bleeding among patients in the aprotinin group than in those receiving the other two study drugs (9.5\% vs $12.1 \%$; relative risk 0.79 , 95\% Cl 0.59-1.05). Aprotinin was associated with a relative increase in 30-day mortality of $>50 \%$ (absolute increase $2.1 \%$ ) compared with the lysine analogs (relative risk 1.53; $95 \% \mathrm{Cl} 1.06-2.22)$, however, and the trial was stopped early.

The authors conclude that, although aprotinin might prevent major bleeding, the unacceptably high mortality risk associated with this agent prohibits its use in patients undergoing high-risk cardiac surgery.

Original article Fergusson DA et al. for the BART Investigators (2008) A comparison of aprotinin and lysine analogues in high-risk cardiac surgery. N Engl J Med 358: 2319-2331 